\title{
Comparison of the efficacy of four drug combinations for immobilization of wild pigs
}

\author{
Christine K. Ellis ${ }^{1}$ (D) $\cdot$ Morgan E. Wehtje ${ }^{2}$ - Lisa L. Wolfe ${ }^{3} \cdot$ Peregrine L. Wolff $^{4} \cdot$ Clayton D. Hilton $^{5} \cdot$ Mark C. Fisher $^{3}$. \\ Shari Green ${ }^{3}$. Michael P. Glow ${ }^{1}$. Joeseph M. Halseth ${ }^{1}$. Michael J. Lavelle ${ }^{1}$. Nathan P. Snow ${ }^{1}$. Eric H. VanNatta ${ }^{1}$. \\ Jack C. Rhyan ${ }^{6} \cdot$ Kurt C. VerCauteren ${ }^{1}$ - William R. Lance ${ }^{7}$. Pauline Nol $^{2}$
}

Received: 24 October 2018 / Revised: 2 August 2019 / Accepted: 15 August 2019 / Published online: 26 August 2019

(C) The Author(s) 2019

\begin{abstract}
Field immobilization of native or invasive wild pigs (Sus scrofa) is challenging. Drug combinations commonly used often result in unsatisfactory immobilization, poor recovery, and adverse side effects, leading to unsafe handling conditions for both animals and humans. We compared four chemical immobilization combinations, medetomidine-midazolam-butorphanol (MMB), butorphanol-azaperone-medetomidine (BAM $\left.{ }^{\mathrm{TM}}\right)$, nalbuphine-medetomidine-azaperone (NalMed-A), and tiletaminezolazepam-xylazine (TZX), to determine which drug combinations might provide better chemical immobilization of wild pigs. We achieved adequate immobilization with no post-recovery morbidity with MMB. Adequate immobilization was achieved with BAM $^{\mathrm{TM}}$; however, we observed post-recovery morbidity. Both MMB and BAM ${ }^{\mathrm{TM}}$ produced more optimal results relative to body temperature, recovery, and post-recovery morbidity and mortality compared to TZX. Adequate immobilization was not achieved with NalMed-A. Of the four drug combinations examined, we conclude that MMB performed most optimally for immobilization and recovery of wild pigs.
\end{abstract}

Keywords Wild pig $\cdot$ Chemical immobilization $\cdot$ Medetomidine $\cdot$ Midazolam $\cdot$ Butorphanol

\section{Introduction}

Capturing and handling wild pigs (Sus scrofa; referred to in the literature as feral swine/pigs/hogs/wild boar/swine, wild swine/ pigs/hogs/boar/boar-feral pig hybrids, Eurasian wild boar, European wild boar, European swine) (Price 1984; Nolte and

Christine K. Ellis

Christine.K.Ellis@aphis.usda.gov

1 USDA-APHIS, Wildlife Services, National Wildlife Research Center, Fort Collins, CO, USA

2 USDA-APHIS - Veterinary Services, Centers for Epidemiology and Animal Health, Fort Collins, CO, USA

3 Colorado Division of Parks and Wildlife, Fort Collins, CO, USA

4 Nevada Department of Wildlife, Reno, NV, USA

5 Cesar Kleberg Wildlife Research Institute, Texas A \& M University, Kingsville, TX, USA

6 Wildlife Pharmaceuticals, Windsor, CO, USA

7 USDA-APHIS - Veterinary Services, National Veterinary Services Laboratory, Fort Collins, CO, USA
Anderson 2015; Keiter et al. 2016; Sweitzer et al. 2016; McCann et al. 2018) for field research focusing on behavior, ecology, movement, or other study objectives present a variety of challenges as such studies typically involve manipulations including attachment of monitoring devices (e.g., radio collars, proximity loggers), sample collection, and translocation, followed by release back into the environment. Development of field research study plans should occur under the guidance of an institutional animal care and use committee (IACUC) and may include a risk analysis to improve study implementation and outcomes (Swart 2004; FASS 2010; Paul et al. 2016). While standard animal research guidelines and regulations may not apply specifically to wildlife species, the basic goals of ethical humane treatment, and limiting stress, distress, discomfort, and pain to the animals must be considered by the IACUC and the research team (William 1959; Smith and Hawkins 2016). The risk analysis should include identification of problems unique to wildlife research such as the inability to accurately assess the age, weight, and health status of wild animals, occurrence of adverse environmental conditions, trapping and release strategies, handling and release stressors, and other study-specific parameters. 
Development of all handling, sampling, capture and release strategies, chemical immobilization, and analgesic regimens should ensure animal and personnel safety. Use of chemical immobilization agents improves efficiency and safety for animal handlers, enhances achievement of study objectives, and decreases stress and other well-described detrimental physiological responses (e.g., hyperthermia, traumatic injury) in wild pigs. Selection of the most appropriate chemical immobilization agents should be based upon the research study objectives, external and internal factors associated with the study, cost, immobilization agent pharmacological effects, immobilization agent availability, safety of animal handlers and the animals, guidance of the IACUC, and the results of the risk analysis.

External (e.g., ambient temperature, environmental conditions, terrain, nearby water bodies or roads, capture techniques, research study objectives) and internal factors (e.g., genetics, gender, age, disease, reproductive status), and the pharmacological effects of the chemical immobilization agents pre-, intra-, and post-immobilization affect the outcome of wild animal immobilization events and research data quality (Wyckoff et al. 2006; Fenati et al. 2008; Barasona et al. 2013). Errors in weight estimation of wild pigs is not uncommon (Fenati et al. 2008) and results in under- or over-dosage of chemical immobilization agents. Over-dosage or administration of supplemental doses of chemical immobilization agents may result in impaired physiological responses (e.g., thermoregulation, respiration, circulation, mentation) during and after sedation, and increased morbidity or death, especially agents that induce prolonged sedation and extended recovery times and have no reversal agent(s) (Gabor et al. 1997; Sweitzer et al. 1997a; Fenati et al. 2008). Post-mortem data are often not collected following wild pig capture or chemical immobilization mortalities; however, capture myopathy, hyperthermia, chemical immobilization, drug-induced cardiovascular collapse, and concomitant physiological or infectious disease are suggested etiologies contributing to wild pig mortalities (Spraker 1982; Wyckoff et al. 2006; Fenati et al. 2008; Barasona et al. 2013; Swindle and Smith 2015). Postimmobilization hyperthermia occurring hours after recovery from chemical immobilization has been reported in domestic swine (Swindle and Smith 2015). It is unknown if this phenomenon occurs in wild pigs; however, it is plausible given this syndrome is associated with elevated blood lactate and stress (Swindle and Smith 2015).

Drugs commonly used to immobilize wild pigs in the field include combinations of tiletamine-zolazepam (TZ) plus xylazine (X) and/or ketamine (K) (Sweitzer et al. 1997a; Plumb 2008; Wyckoff et al. 2009; Barasona et al. 2013). Tiletamine is a cyclohexamine compound that elicits central nervous system dissociation, visceral analgesia, and superficial anesthesia (Plumb 2008). Adverse effects may include muscle hypertonia and rigidity, tachycardia, vomiting, hypertension, and erratic/prolonged recovery (Plumb 2008;
Carpenter 2013). Zolazepam is a benzodiazepine drug that induces sedation, and has anxiolytic and muscle relaxant properties; however, re-sedation can occur in animals receiving large or multiple doses of zolazepam. Xylazine is an $\alpha 2$ adrenergic agonist that provides sedation, visceral analgesia, and muscle relaxation that may be reversed with $\alpha 2$ adrenergic receptor antagonists such as atipamezole, tolazoline, and yohimbine (Plumb 2008; Barasona et al. 2013). Ketamine is a cyclohexamine drug with rapid onset of action (1-4 min) and short duration of action (12$25 \mathrm{~min}$ ). Important adverse effects include hyperthermia, emesis, and muscle hypertonia and rigidity. Potentiation of the pharmacological effects of butorphanol and other opiates can occur with this agent (Plumb 2008). Reversal agents are not available for tiletamine or ketamine. The duration of action and time to full recovery associated with $\mathrm{TZ}$ combinations are long (tiletamine, 60-90 min; zolazepam, 1-5.5 h) in most animal species, including swine (Plumb 2008; Kumar et al. 2014). Use of TZ in domestic and wild pigs has been observed to elicit prolonged, rough recoveries that include traumatic behaviors (e.g., paddling, struggling to achieve sternal recumbency or standing, staggering, collision with solid objects), which may contribute to post-immobilization persistence of hyperthermia, and the pathology of capture myopathy (Gabor et al. 1997; Sweitzer et al. 1997a, 1997b; Enqvist 2000; Wyckoff et al. 2006; Kim et al. 2007; Fenati et al. 2008; Barasona et al. 2013).

The health and safety of animal handlers and study subjects during handling events is important, especially when handling large aggressive animals such as wild pigs. Use of appropriate animal handling systems that minimize stress and injury to the animals, and maximize human safety are critical. Efficacious chemical immobilization agents with excellent immobilization attributes, wide margins of safety, that result in good sedation, quiet expedient recovery, and few prolonged postrecovery sequelae are important adjuncts to the handling process. Drugs with prolonged times to full recovery and deleterious post-recovery effects may affect animal behavior and responses well beyond the time animals are perceived to be "recovered" and released back to the environment. Additionally, immobilization drugs and their metabolites can persist in tissues for long periods of time (Swindle and Smith 2015) and as such may pose health risks to hunters.

It is of paramount importance to identify the most effective tools available to minimize wildlife capture and handling risks. This includes identification of chemical immobilization agents that offer alternatives to the use of TZ, TZX, TKX, and TZKX in wild pigs. Ideally, these alternatives would provide improved deep sedation, muscle relaxation, adequate analgesia, capability for reversal, short duration of quiet recovery and post-recovery behaviors, and discourage induction or exacerbation of adverse physiological responses (e.g., hyperthermia, capture myopathy). Two drug combinations used 
successfully in other wildlife species include butorphanolazaperone-medetomidine (BAM ${ }^{\mathrm{TM}}$ ) and nalbuphineazaperone-medetomidine (NalMed-A) which have proved effective for immobilization of black bear (Ursus americanus), elk (Cervus canadensis), bison (Bison bison), and ibex (Capra nubiana) (Mich et al. 2008; Wolfe et al. 2008; Miller et al. 2009; Wolfe et al. 2014a, 2014b, 2017; Wolfe et al., 2014a, b; Lapid and Shilo-Benjamini 2015). Medetomidine-midazolam-butorphanol (MMB) is a drug combination used successfully for sedation of domestic and pet pigs in veterinary medicine (Wolff 2009). Butorphanol is an opiate partial agonist with analgesic and sedative properties and few adverse effects. Onset of action is rapid, with peak effect occurring 15-30 min after administration, and duration of action lasting up to $4 \mathrm{~h}$. Pharmacological effects of this drug are potentiated when administered with ketamine (Plumb 2008). Azaperone, a butyrophenone tranquilizer, produces sedation but no analgesia and has been demonstrated to prevent the development or exacerbation of malignant hyperthermia in domestic pigs. Onset of action is rapid (5-10 $\mathrm{min}$ ), and duration of action ranges from 2 to $4 \mathrm{~h}$ (Plumb 2008). Medetomidine is an $\alpha 2$-adrenergic agonist that elicits sedation, anxiolysis, and analgesia. Adverse effects include bradycardia, decreased respiration, hypothermia, and rarely, paradoxical excitation and apnea (Plumb 2008). Advantages include capability to reverse with atipamezole. Midazolam is a benzodiazepine that produces sedation, anxiolysis, and muscle relaxation. Adverse reactions are primarily limited to respiratory depression. Duration of action lasts an average of $2 \mathrm{~h}(1-6 \mathrm{~h}$ range) and is species variable (Plumb 2008; Carpenter 2013). Nalbuphine is an opioid partial agonist that produces sedation and limited analgesia. Severe respiratory depression may occur when this drug is used in combination with other central nervous system or respiratory depressant agents. Advantages include the capability to reverse with naloxone (Plumb 2008).

Here, we describe an exploratory study to assess and compare the efficacy of four drug combinations, MMB, BAM ${ }^{\mathrm{TM}}$, NalMed-A, and TZX, for wild pig immobilization. In addition, we measured levels of detectible drug in edible tissues at multiple time points to monitor drug residues in this hunted species.

\section{Methods}

Twenty-three captive-raised wild pigs (female $=13$, male $=10$; offspring of wild pigs collected in Texas, USA) were used for this study. Animals ranged in age from 5 to 7 months and weights ranged from 12.27 to $30.00 \mathrm{~kg}$ at the beginning of phase I and 16.35-43.64 kg during phase IV of the study. Pigs were housed at the United States Department of Agriculture (USDA)-Animal and Plant Health Inspection Service (APHIS)/Colorado State University (CSU) Wildlife
Research Facility, Fort Collins, Colorado, USA $\left(40^{\circ} 34^{\prime} 5^{\prime \prime} \mathrm{N}\right.$, $105^{\circ} 08^{\prime} 49^{\prime \prime} \mathrm{W}$; elevation approximately $1519 \mathrm{~m}$ ). Animals were held in $3 \mathrm{~m} \times 13 \mathrm{~m}$ solid panel runs with soil substrate, and were offered a commercial swine ration fed at $0.5 \mathrm{~kg} / \mathrm{animal} /$ day and water ad libitum. The study plan was approved by the CSU Animal Care and Use Committee (IACUC 17-7386A) and the USDA-APHIS, Wildlife Services, National Wildlife Research Center, Animal Care and Use Committee (QA-2803).

Medetomidine $(40 \mathrm{mg} / \mathrm{mL})$, midazolam $(50 \mathrm{mg} / \mathrm{mL})$, butorphanol $(30 \mathrm{mg} / \mathrm{mL})$, and atipamezole $(25 \mathrm{mg} / \mathrm{mL})$ were obtained individually, and BAM ${ }^{\text {TM }}$ (butorphanol, $27.3 \mathrm{mg} /$ $\mathrm{mL}$; azaperone, $9.1 \mathrm{mg} / \mathrm{mL}$; medetomidine, $10.9 \mathrm{mg} / \mathrm{mL}$ ) and NalMed-A (nalbuphine, $40 \mathrm{mg} / \mathrm{mL}$; azaperone, $10 \mathrm{mg} /$ $\mathrm{mL}$; medetomidine, $10 \mathrm{mg} / \mathrm{mL}$ ) were obtained as premixed products from Wildlife Pharmaceuticals Inc., Windsor, Colorado, USA. Tiletamine-zolazepam (Telazol® (tiletamine $50 \mathrm{mg} / \mathrm{mL}$, zolazepam $50 \mathrm{mg} / \mathrm{mL}$ )) was obtained from Zoetis (Parsippany, New Jersey, USA) and xylazine (AnaSed ${ }^{\circledR}$ $(100 \mathrm{mg} / \mathrm{mL})$ from Lloyd Inc. (Shenandoah, Iowa, USA).

The study was conducted in four phases, each separated by a 2-week wash-out period. The first and second phases were used to optimize and test BAM ${ }^{\mathrm{TM}}$ and NalMed-A dosages, respectively. The third phase was used to optimize and test MMB dosages and to collect edible tissues from six test subjects. During the fourth phase, six wild pigs were immobilized with an established TZX dosage (TZ, $4.40 \mathrm{mg} / \mathrm{kg}$; X, $2.5 \mathrm{mg} / \mathrm{kg}$ ) (Ko et al. 1993; Sweitzer et al. 1997a, 1997b; Enqvist 2000; Plumb 2008; Carpenter 2013) to obtain comparative pre-, intra-, and post-immobilization data and tissues for residue testing, and six animals were immobilized with $\mathrm{BAM}^{\mathrm{TM}}$ to obtain tissue residue samples.

Pigs were randomized into three treatment groups and were fasted for $24 \mathrm{~h}$ prior to immobilization. Each animal was weighed and manually restrained with a mechanical squeeze in a pig-specific handling box $(46.4 \mathrm{~cm} \times 99.1 \mathrm{~cm} \times$ $68.58 \mathrm{~cm}$ ). All drugs were delivered by hand injection intramuscularly (IM) deep into the lateral thigh using a $3.0-\mathrm{mL}$ syringe and $18 \mathrm{G} \times 2 \mathrm{in}$. hypodermic needle. Immediately after injection, restraint was removed and the handling box covered to minimize stimulation. Each wild pig was monitored for changes in behavior and levels of sedation. A dosage was considered adequate for sedation when immobilization occurred within $20 \mathrm{~min}$, and allowed safe manipulation of the animal and measurement of physiological parameters. Once optimized dosages were determined, pigs remaining in the respective treatment groups were chemically immobilized and physiological parameters monitored.

The optimization procedure for BAM ${ }^{\mathrm{TM}}$ and NalMed-A occurred as follows: The recommended starting dosage in wild pigs for BAM ${ }^{\mathrm{TM}}(1.0 \mathrm{~mL} / 45 \mathrm{~kg} \mathrm{IM}$; equivalent to butorphanol $0.60 \mathrm{mg} / \mathrm{kg}$, azaperone $0.20 \mathrm{mg} / \mathrm{kg}$, and medetomidine $0.22 \mathrm{mg} / \mathrm{kg}$ ) or NalMed-A ( $1.5 \mathrm{~mL} / 45 \mathrm{~kg} \mathrm{IM}$; equivalent to nalbuphine $1.3 \mathrm{mg} / \mathrm{kg}$, medetomidine 
$0.33 \mathrm{mg} / \mathrm{kg}$, and azaperone $0.33 \mathrm{mg} / \mathrm{kg}$ ) were drawn directly into a syringe and administered to the first wild pig. If sedation for safe handling occurred within $20 \mathrm{~min}$, the animal was removed from the restraint box, eye lubrication ointment (Paralube ${ }^{\circledR}$ Vet Ointment; Dechra Pharmaceuticals, Quebec, Canada) was placed in each eye, a blindfold was applied, and the animal was handled and monitored, followed by IM administration of the antagonist drug (atipamezole $5.0 \mathrm{mg}$ per $1.0 \mathrm{mg}$ medetomidine administered). The animal was then transferred to a recovery pen for recovery monitoring. A second wild pig was restrained as described above and administered a $10 \%$ decreased dosage of the candidate drug combination and observed for response. If this animal became adequately sedated, it was monitored and recovered as described above. Subsequent animals would then be administered $10 \%$ decreasing dosages of the candidate drug combination until adequate sedation could not be achieved. If the first wild pig was inadequately sedated (safe handling not achieved $20 \mathrm{~min}$ post-injection), it was administered the appropriate reversal antagonist, moved to the recovery pen, and monitored until full recovery. The second wild pig was subsequently administered a $20 \%$ increased dosage of the candidate drug combination and observed. This process would be repeated until an adequate level of sedation was achieved. The next pig would receive a $10 \%$ decreased dosage of the candidate drug combination to complete the drug optimization process.

MMB is not available commercially as a premixed drug combination, requiring optimization of each drug in the combination. The starting dosage for each drug was calculated using previously published dosages for each drug component (e.g., medetomidine $0.06 \mathrm{mg} / \mathrm{kg}$; midazolam $0.3 \mathrm{mg} / \mathrm{kg}$; butorphanol $0.3 \mathrm{mg} / \mathrm{kg}$ ) (Wolff 2009). Each drug was individually withdrawn from a stock bottle using separate syringes and combined into one syringe for IM injection. If adequate sedation was achieved, the animal was removed from the pig handler and processed as described above, followed by administration of the antagonist drug (atipamezole $5.0 \mathrm{mg}$ for every $1.0 \mathrm{mg}$ medetomidine administered) and recovery monitoring. Dosage adjustments for midazolam and butorphanol were changed in $10 \%$ or $20 \%$ increments as described above. The concentration of medetomidine was such that dosage adjustments were made in increments of $0.01 \mathrm{~mL}(0.04 \mathrm{mg})$.

Prior to administration of TZX, the two drug components were drawn up individually then combined into one syringe used for IM injection. Animals immobilized with TZX were administered tolazoline $\mathrm{HCl}$ (Tolazine ${ }^{\circledR}$; Lloyd Inc.), $2.2 \mathrm{mg} / \mathrm{kg}$ to antagonize xylazine.

\section{Parameters}

Induction was measured as time ( $\mathrm{min}$ ) from drug administration to level 2 (partial sedation, ataxia), level 3 (partial sedation, sternal recumbency), and level 4 induction (lateral recumbency, complete sedation). Chemically immobilized wild pigs reaching level 4 sedation adequate for handling 20 min post-IM injection were monitored physiologically (e.g., rectal temperature $(\mathrm{T})$, heart rate $(\mathrm{HR}$, beats $/ \mathrm{min})$, respiratory rate $(\mathrm{R}$, breaths $/ \mathrm{min})$, blood pressure $(\mathrm{BP})$, peripheral capillary oxygen saturation $\left.\left(\mathrm{SpO}_{2}\right)\right)$, and adequacy of sedation was evaluated (e.g., $1=$ poor through $5=$ best) by assessing muscle rigidity, jaw tone, and response to field handling procedures (e.g., loud noise; body repositioning; blood collection; ear tag placement).

Monitoring time began when animals reached level 4 sedation adequate for handling $20 \mathrm{~min}$ post-IM injection and continued for $40 \mathrm{~min}$ (average time to work-up wild pigs in the field) or $60 \mathrm{~min}$ post-injection (two randomly selected individuals per treatment group) to assess the potential duration of immobilization. At the end of the monitoring period, all wild pigs were administered the appropriate antagonist drug, placed in a recovery pen, and monitored until full recovery was documented. Recovery was measured as the time (min) from antagonist administration to each level of recovery (e.g., level 2, increased respiration; level 3, able to hold head up; level 4, standing). Post-recovery behaviors were observed until animals exhibited normal behaviors and appeared to be experiencing no overt residual effects of the immobilization agents.

Rectal temperature (T), HR, R, and $\mathrm{SpO}_{2}$ were monitored and recorded every $5 \mathrm{~min}$. A handheld pulse oximeter (Rad57; Masimo, Irvine, California, USA) and tongue sensor were used to measure $\mathrm{SpO}_{2}$. Nasal insufflation of supplemental oxygen was initiated at $3 \mathrm{~L} / \mathrm{min}$ if $\mathrm{SpO}_{2}$ levels fell below $90 \%$. Blood pressure was measured with an automated digital blood pressure monitor (CONTEC08A Sphygmomanometer; Contec Medical Systems Co., Ltd, Qinhuangdao, China) and 10-19 mm pediatric cuff placed over the ulnar artery proximal to the carpal joint (Hodgkin et al. 1982).

\section{Tissue drug residues}

Two wild pigs immobilized with MMB were euthanized during phase III of the study 3,5 , and 7 days post-immobilization and edible tissues (e.g., fat, liver, kidney, semitendinosis/ semimembranosis muscle) were collected to determine drug residue levels (limit of detection $0.01 \mathrm{ppm}$ ). No wild pigs immobilized with BAM ${ }^{\mathrm{TM}}$ during phase I were euthanized in order to preserve adequate numbers of pigs throughout phases II and III. Instead, during phase IV, six individuals were immobilized with $\mathrm{BAM}^{\mathrm{TM}}$ and euthanized at the above-stated time-points, as were the six TZX-immobilized animals. Samples were stored at $-80{ }^{\circ} \mathrm{C}$ until analysis by liquid chromatography-tandem mass spectrometry (LC-MS/ MS; Texas Veterinary Medical Diagnostic Laboratory, Texas A\&M University, College Station, Texas, USA). 


\section{Assessment of measured parameters}

Physiological parameters were evaluated observationally to assess changes occurring over the course of the chemical immobilization period for TZX and each successfully optimized candidate chemical immobilization combination. Recorded observations were compared to published normal values for domestic and wild pigs (Sakaguchi et al. 1992; Ko et al. 1993; Sweitzer et al. 1997a; Williams et al. 2002; Plumb 2008; Carpenter 2013; Swindle and Smith 2015). Prior to initiating statistical analyses, we visually assessed all data for normality to determine the most suitable statistical methodology. Overall mean HR, R, and $\mathrm{T}$ data and residuals appeared normally distributed, and we used linear mixed models to assess the effect of drug combinations on these parameters. For each response variable, we used a fixed effect to describe the effect of treatment group and sex. Range in weight was controlled for by including weight as a random effect. We also accounted for the random effect of individual in all models to account for any variability between individual animals. The effect of each drug combination on induction time to each level of sedation and time from administration of antagonist to recovery level 4 were not normally distributed and were analyzed using a Kruskal-Wallis test. We carried out all statistical analyses in the $\mathrm{R}$ programming environment (http://www.R-project.org) using the linear mixed model package lme4 and lmerTest.

\section{Results}

\section{Drug optimization}

Two drug combinations (MMB and BAM ${ }^{\mathrm{TM}}$ ) were successfully optimized in wild pigs (Table 1). The optimized BAM ${ }^{\mathrm{TM}}$ dosage was higher $(1.2 \mathrm{~mL} / 45 \mathrm{~kg})$ than the suggested starting dosage, and individual drug dosages for butorphanol and medetomidine were greater than the published recommended dosage ranges for swine (Sakaguchi et al. 1992; Plumb 2008; Carpenter 2013; Swindle and Smith 2015). The optimized individual MMB drug dosages all fell within recommended dosage ranges for swine (Sakaguchi et al. 1992; Plumb 2008; Carpenter 2013; Swindle and Smith 2015). Wild pigs administered optimized dosages of MMB and BAM ${ }^{\mathrm{TM}}$ remained safely immobilized until antagonist administration, including the two animals monitored for $60 \mathrm{~min}$.

An effective NalMed-A dosage could not be determined. The recommended dosage was incrementally increased to $2.0 \mathrm{~mL} / 45 \mathrm{~kg}$, which proved inadequate to produce a level of sedation amenable to safe handling. Further increases in dosage were deemed infeasible for safe or practical use. Wild pigs administered NalMed-A were observed to reach sedation levels 2,3 , and 4 at 7-15 $\mathrm{min} ; 10-25 \mathrm{~min}$; and 16$37 \mathrm{~min}$, respectively, but recovered quickly to standing when stimulated by minor manipulations (e.g., taking $\mathrm{T}$, touching the face, removing the animal from the handler). When animals were moved to the recovery pens, they were often standing and fully mobile.

\section{Induction and sedation quality}

Observationally, the average time to level 2 sedation for MMB and $\mathrm{BAM}^{\mathrm{TM}}$-treated wild pigs (3.8 $\mathrm{min}$ and $4.1 \mathrm{~min}$, respectively) were similar to the time noted in one TZX animal (4.5 min; the other TZX-treated wild pigs progressed directly to level 3) (Table 2). The average times to induction level 3 were similar for MMB and BAM ${ }^{\mathrm{TM}}(8.1 \mathrm{~min}$ and $7.8 \mathrm{~min}$, respectively) and longer than that observed for TZX (4.0 min). Animals administered TZX reached level 4 induction faster $(8.6 \mathrm{~min})$ than those sedated with MMB (12.7 min) or BAM ${ }^{\mathrm{TM}}$ (11.6 min). Statistically, time from administration of MMB, BAM ${ }^{\mathrm{TM}}$, and TZX to sedation levels 2 and 4 were not significantly different; however, time to reach level 3 was statistically different (Kruskal-Wallis test $p=0.02$ ) in the MMB versus TZX, and BAM ${ }^{\mathrm{TM}}$ versus TZX comparisons. There was no statistical difference in time to reach level 3 between $\mathrm{BAM}^{\mathrm{TM}}$ and MMB.

Good sedation quality was observed for MMB, BAM ${ }^{\mathrm{TM}}$, and TZX. No responses were noted in any immobilized animals when they were removed from the restraint box, during application of the $\mathrm{SpO}^{2}$ monitor, or during assessment of $\mathrm{T}$, $\mathrm{HR}, \mathrm{R}$, and BP throughout the monitoring period. Jaw tone was comparatively relaxed, and no responses were observed to loud noise, venipuncture, or when minor body repositioning (e.g., manipulating legs, placing in lateral recumbency) occurred across all treatment groups; however, when $\mathrm{BAM}^{\mathrm{TM}}$-immobilized wild pigs were placed in dorsal recumbency to mimic being weighed or transported without use of a stretcher, we did observe vocal and movement responses in some animals. Observationally, muscle rigidity was greater during the first $15 \mathrm{~min}$ of the monitoring period when animals were immobilized with TZX versus MMB and BAM ${ }^{\mathrm{TM}}$. Reaction to ear tagging (i.e., ear flick) was observed in 5/14 (35\%) of BAM ${ }^{\mathrm{TM}}, 2 / 14$ (14\%) of MMB, and 1/6 (17\%) TZXtreated wild pigs.

Statistically, MMB and BAM ${ }^{\mathrm{TM}}$ did not differ in their effects on overall mean T, HR, and R while TZX differed significantly in all three parameters from both MMB $(p \leq$ $0.00001)$ and $\operatorname{BAM}^{\mathrm{TM}}(p \leq 0.00513)$. Variation among individuals was high for all combinations, and males generally had slightly but not significantly lower T, HR, and R values than females. Weight did not account for any variation in the model for $\mathrm{T}$, was equal to individual variation in the model for $\mathrm{R}$, and accounted for most of the variation among individuals in the model examining HR. Statistical analyses were not performed on $\mathrm{SpO}_{2}$ or blood pressure. 
Table 1 Drugs and drug combinations evaluated for use as chemical immobilization agents used in captive wild pigs (Sus scrofa) during comparison of immobilization drugs medetomidine, midazolam, butorphanol (MMB); butorphanol, azaperone, medetomidine (BAM $\left.{ }^{\mathrm{TM}}\right)$; and tiletamine-zolazepam, xylazine (TZX)

\begin{tabular}{lllll}
\hline Drug combination & $\begin{array}{l}\text { Optimized } \\
\text { drug dosage }\end{array}$ & Individual drugs & $\begin{array}{l}\text { Individual drug dosages } \\
\text { in combination }(\mathrm{mg} / \mathrm{kg})\end{array}$ & $\begin{array}{l}\text { Published dosage } \\
\text { ranges }{ }^{\mathrm{a}}(\mathrm{mg} / \mathrm{kg})\end{array}$ \\
\hline $\mathrm{MMB}$ & $0.06 \mathrm{mg} / \mathrm{kg}$ & Medetomidine & 0.06 & $0.03-0.15$ \\
& $0.30 \mathrm{mg} / \mathrm{kg}$ & Midazolam & 0.30 & $1.00-10.00$ \\
& $0.30 \mathrm{mg} / \mathrm{kg}$ & Butorphanol & 0.30 & $0.10-0.50$ \\
$\mathrm{BAM}^{\mathrm{TM}}$ & $1.2 \mathrm{~mL} / 45 \mathrm{~kg}$ & Butorphanol & 0.72 & $0.10-0.50$ \\
& & Azaperone & 0.24 & $0.15-0.30$ \\
& \multirow{2}{*}{$\mathrm{NZX}$} & Medetomidine & 0.27 & $0.03-0.15$ \\
& & Tiletamine-zolazepam & 4.40 & $2.0-4.4$ \\
& & Xylazine & 2.50 & $2.0-2.50$ \\
\hline
\end{tabular}

NA not applicable

${ }^{\text {a }}$ Sakaguchi et al. 1992; Ko et al. 1993; Sweitzer et al. 1997a; Plumb 2008; Carpenter 2013; Swindle and Smith 2015

Observationally, the mean $\mathrm{T}$ recorded in $\mathrm{MMB}$ and $\mathrm{BAM}^{\mathrm{TM}}$-treated wild pigs at the beginning of the monitoring periods were within the normal $\mathrm{T}$ range reported for swine (Kahn et al. 2005; Carpenter 2013; Morgaz et al. 2015) and decreased to slightly below normal (Table 3 ). The mean $\mathrm{T}$ of TZX-treated animals was above the upper limit of $\mathrm{T}$ normal at the initiation of monitoring, and exceeded or remained in the high normal range throughout the monitoring period.

Individually, the observed T of 5/14 (36\%) MMB- and 2/14 (14\%) BAM ${ }^{\mathrm{TM}}$-immobilized wild pigs 20 min post-drug administration approached or exceeded the normal T range; however, all temperatures fell within or slightly below normal during continued monitoring. The $\mathrm{T}$ of $2 / 6$ (33\%) TZX-treated individuals were above normal at the initiation of monitoring and remained elevated, necessitating topical application of cool water and alcohol, and cool water enemas were administered to one individual that remained refractory to topical treatment. Temperatures of the other TZX-treated animals were at the upper limit of normal $\mathrm{T}$ at the initiation of monitoring and

Table 2 Observed times to levels of induction and recovery for captive wild pigs (Sus scrofa) during comparison of immobilization drugs medetomidine, midazolam, butorphanol (MMB); butorphanol, azaperone, medetomidine (BAM $\left.{ }^{\mathrm{TM}}\right)$; and tiletamine-zolazepam, xylazine (TZX)

\begin{tabular}{llll}
\hline & \multicolumn{2}{l}{ Drug combinations } \\
\cline { 2 - 4 } $\begin{array}{llll}\text { Induction times } \\
\text { (mean min } \pm \mathrm{SD})\end{array}$ & $\begin{array}{l}\text { MMB } \\
(N=14)\end{array}$ & $\begin{array}{l}\mathrm{BAM}^{\mathrm{TM}} \\
(N=14)\end{array}$ & $\begin{array}{l}\mathrm{TZX} \\
(N=6)\end{array}$ \\
\hline Level 2 (ataxia) & $3.8 \pm 0.6$ & $4.1 \pm 1.5$ & $4.5^{1}$ \\
Level 3 (sternal recumbency) & $8.1 \pm 2.1 \mathrm{a}$ & $7.8 \pm 3.5 \mathrm{a}$ & $4.0 \pm 1.4 \mathrm{~b}$ \\
Level 4 (lateral recumbency) & $12.7 \pm 1.5$ & $11.6 \pm 5.4$ & $8.6 \pm 3.3$ \\
Time to recovery & & & \\
Level 4 (standing) & $7.5 \pm 4.8$ & $6.7 \pm 3.5$ & $10.9 \pm 7.0$ \\
\hline
\end{tabular}

Values with different letters are significantly different from each other

${ }^{1}$ Only one individual. All others progressed directly to level 3 remained higher than the T recorded for these individuals during $\mathrm{MMB}$ and $\mathrm{BAM}^{\mathrm{TM}}$ sedation.

The mean HR of wild pigs immobilized with MMB and BAM $^{\mathrm{TM}}$ were similar and remained within normal HR limits throughout the monitoring periods. By comparison, during TZX immobilization, mean HR were observed to be higher in all individuals, yet remained within normal limits. Mean $\mathrm{R}$ were within normal limits when wild pigs were immobilized with MMB and BAM ${ }^{\mathrm{TM}}$ and TXZ; however, R tended to be lower throughout the monitoring periods when animals were administered MMB and BAM ${ }^{\mathrm{TM}}$.

All wild pigs required administration of supplemental oxygen regardless of the chemical immobilization agents used. Mean $\mathrm{SpO}_{2}$ measurements recorded without oxygen supplementation at the initiation of monitoring were $90 \%, 87 \%$, and $91 \%$ for MMB, BAM ${ }^{\mathrm{TM}}$, and TZX, respectively. These values recovered post-oxygen supplementation to mean measured saturation levels of $93 \%$ (MMB), 93\% (BAM $\left.{ }^{\mathrm{TM}}\right)$, and $93 \%$ (TZX) at $40 \mathrm{~min}$ and $93 \%$ (MMB), 90\%, and 98\% (TZX) at $60 \mathrm{~min}$. We were unable to consistently measure BP in all animals at all time-points; however, when measured, systolic and diastolic BP were within normal limits based on published values (Hodgkin et al. 1982; Gianotti et al. 2010).

\section{Recovery}

Average times to recovery level 4 after administration of appropriate reversal agents were observed to be slightly shorter when wild pigs were immobilized with $\mathrm{MMB}$ and BAM ${ }^{\mathrm{TM}}$ (7.5 $\mathrm{min}$ and $6.7 \mathrm{~min}$, respectively) versus TZX (10.9 min) (Table 2). Observationally, recovery quality following MMB and $\mathrm{BAM}^{\mathrm{TM}}$ immobilization was improved over recoveries observed following TZX immobilization. After administration of atipamezole, wild pigs immobilized with MMB and BAM $^{\mathrm{TM}}$ quietly transitioned from lateral recumbency to sternal recumbency or standing, then walked away or stood quietly and were capable of coordinated purposeful movements. 
Table 3 Range of physiological parameters measured in captive wild pigs (Sus scrofa) during comparison of immobilization drugs medetomidine, midazolam, butorphanol (MMB) and butorphanol, azaperone, medetomidine $\left(\mathrm{BAM}^{\mathrm{TM}}\right)$ to tiletamine-zolazepam/xylazine (TZX)

\begin{tabular}{llllll}
\hline Drug protocol & & Temperature $\left({ }^{\circ} \mathrm{C}\right)$ & $\begin{array}{l}\text { Heart rate } \\
\text { (beats } / \\
\text { min) }\end{array}$ & $\begin{array}{l}\text { Respiratory rate } \\
\text { (breaths/min) }\end{array}$ & $\begin{array}{l}\text { Blood pressure } \\
(\mathrm{mm} \mathrm{Hg})^{*}\end{array}$ \\
\hline Normal* & Minimum & 38.85 & 50 & 10 & $152 / 109$ \\
& Maximum & 39.80 & 100 & 20 & $230 / 165$ \\
$\operatorname{MMB}(N=14)$ & Minimum & 36.80 & 48 & 16 & $108 / 54$ \\
& Maximum & 40.30 & 104 & 70 & $191 / 128$ \\
$\operatorname{BAM}^{\mathrm{TM}}(N=14)$ & Minimum & 35.60 & 38 & 16 & $122 / 50$ \\
& Maximum & 41.30 & 120 & 82 & $220 / 129$ \\
$\operatorname{TZX}(N=6)$ & Minimum & 38.10 & 60 & 36 & $110 / 52$ \\
& Maximum & 41.10 & 116 & 78 & $203 / 122$ \\
\hline
\end{tabular}

*Hodgkin et al. 1982; Hannon et al. 1989; Plumb 2008; Gianotti et al. 2010; Sipos et al. 2013; Morgaz et al. 2015
Complete recovery from immobilization was observed in all MMB animals and all but two $\mathrm{BAM}^{\mathrm{TM}}$-treated individuals an average of $2 \mathrm{~h}$ after standing. By comparison, the recoveries observed in wild pigs following TZX immobilization were rough. During progression from lateral recumbency to standing, observed behaviors in all animals included paddling, struggling to rise, falling over, ataxia, and incoordination, and attempts to run followed by falling to the ground or against the sides of the recovery area. These behaviors and muscle rigidity were observed in some individuals for prolonged periods (45-90 $\mathrm{min}$ ).

\section{Morbidity and mortality}

None of the pigs in the MMB group appeared to experience behaviors or actions post-immobilization that might contribute to morbidity and there were no mortalities. Two BAM ${ }^{\mathrm{TM}}$ treated pigs (14\%) were observed vomiting, drooling, and appeared depressed post-recovery. One animal appeared fully recovered after $3 \mathrm{~h}$, while the other remained depressed, anorexic, and sternally recumbent for $24 \mathrm{~h}$ before appearing fully recovered. All animals immobilized with TZX experienced rough recoveries and long post-recovery periods (i.e., $90 \mathrm{~min}$ ) that likely contributed to post-immobilization trauma, stress, and morbidity. One animal exhibited symptoms of hyperthermia (i.e., panting) during recovery but was too fractious to be re-captured for veterinary evaluation or intervention. A second animal began panting, became laterally recumbent, and had a recorded $\mathrm{T}$ of $40.6{ }^{\circ} \mathrm{C}$ that returned to normal limits after external application of alcohol and water. This individual remained in lateral recumbency for $3 \mathrm{~h}$, developed mucoid bloody diarrhea, and died. Significant gross necropsy findings included sub-endothelial hemorrhage in the ventricular walls of the heart; a 4- $\mathrm{mm}^{2}$ vegetative lesion on the mitral valve, and submucosal hemorrhage in the wall of the stomach and duodenum. Histopathological findings were consistent with vegetative endocarditis, recent aspiration of ingesta, prolonged hypoxia, and hypotensive shock.

\section{Tissue residues}

No drug residues were found in any tissues collected from pigs at 3 and 5 days post-MMB administration, but medetomidine and butorphanol residues were identified in fat collected from one animal 7 days after immobilization (Table 4). Azaperone was found in the muscle tissues of one pig 3 days after BAM ${ }^{\mathrm{TM}}$ administration, and azaperone or butorphanol were identified in the tissues (muscle and fat, respectively) of the two pigs euthanized 7 days post-BAM ${ }^{\mathrm{TM}}$ administration. Drug residues found in the tissues of the two pigs euthanized 3 days after TZX sedation included zolazepam (one pig; muscle and fat) and xylazine (both pigs; liver, kidney). Xylazine residues were found in liver and kidney tissues collected from one pig euthanized at day 5 postTZX sedation, and tiletamine (muscle), zolazepam (muscle, fat), and xylazine (muscle, liver, kidney) residues were detected in tissues from the other day 5 TZX-treated pig. Xylazine residues were identified in the liver and kidney tissues in one TZX pig euthanized 7 days after treatment.

\section{Discussion}

The optimized dosage of MMB provided good chemical immobilization and the most desirable recovery for wild pigs in this study. Although mean induction time was 4.1 min longer than that of TZX, this induction period is acceptable for both captive and field use. Body temperatures decreased over the course of the monitoring period, even in five wild pigs with initial Ts that approached or exceeded normal published $\mathrm{T}$ ranges and received no interventive treatment. Heart and $\mathrm{R}$ rates were within published normal limits, and the only responses to manipulations simulating field work occurred during ear tagging in five individuals (i.e., ear flick). Recoveries were smooth and rapid, and few observable behaviors that might exacerbate morbidities were observed. Animals remained quiet, but cognizant, capable of purposeful movement, and able to interact with other members 
Table 4 Drug residues detected in selected tissues collected from wild pigs (Sus scrofa) on days 3, 5, and 7 post-administration of medetomidinemidazolam-butorphanol (MMB); butorphanol-azaperone-medetomidine (BAM $\left.{ }^{\mathrm{TM}}\right)$; or tiletamine/zolazepam-xylazine (TZX)

\begin{tabular}{|c|c|c|c|c|c|c|}
\hline Drug combination & Animal & Days post-immobilization & Muscle & Fat & Liver & Kidney \\
\hline \multirow[t]{6}{*}{$\mathrm{BAM}^{\mathrm{TM}}$} & 1 & 3 & Azaperone & ND & $\mathrm{ND}$ & ND \\
\hline & 2 & 3 & ND & ND & ND & ND \\
\hline & 3 & 5 & ND & ND & $\mathrm{ND}$ & ND \\
\hline & 4 & 5 & ND & ND & ND & ND \\
\hline & 5 & 7 & Azaperone & ND & ND & ND \\
\hline & 6 & 7 & ND & Butorphanol & ND & ND \\
\hline \multirow[t]{6}{*}{ MMB } & 1 & 3 & ND & ND & ND & ND \\
\hline & 2 & 3 & ND & ND & ND & ND \\
\hline & 3 & 5 & ND & ND & ND & ND \\
\hline & 4 & 5 & ND & ND & ND & ND \\
\hline & 5 & 7 & ND & ND & ND & ND \\
\hline & 6 & 7 & ND & Medetomidine Butorphanol & ND & ND \\
\hline \multirow[t]{5}{*}{ TZX } & 1 & 3 & ND & ND & Xylazine & Xylazine \\
\hline & 2 & 3 & Zolazepam & Zolazepam & Xylazine & Xylazine \\
\hline & 3 & 5 & ND & ND & Xylazine & Xylazine \\
\hline & 4 & 5 & Tiletamine Zolazepam Xylazine & Zolazepam & Xylazine & Xylazine \\
\hline & 5 & 7 & ND & ND & Xylazine & Xylazine \\
\hline
\end{tabular}

$N D$ not detected

of their respective group post-recovery. There are multiple advantages relative to the use of MMB for chemical immobilization of wild pigs that are to be released after capture. The individual drugs in the optimized combination are within published dosage ranges (Sakaguchi et al. 1992; Ko et al. 1993; Plumb 2008; Carpenter 2013; Swindle and Smith 2015) allowing for supplemental drug administration in the field, and relative safety if overdosage occurs. In addition to the good induction, sedation, and recovery quality, all three drugs in the combination provide analgesia and muscle relaxation. The highly sedative effects of medetomidine are reversible with atipamezole, allowing animals to continue to benefit from the analgesic, anxiolytic, and sedative effects of butorphanol and midazolam for 2-4 $\mathrm{h}$. During this interval, animals are still capable of purposeful responses to stimuli. Disadvantages of MMB include the possibility of bradycardia with/without arrhythmia, the persistence of primary or secondary drug metabolites in tissues, and the requirement for controlled substance documentation in some countries. Additionally, this drug combination requires compounding to achieve concentrations optimal for combination, which in turn can lead to limited availability and increased cost.

Desirable induction, monitoring, recovery, and physiological outcomes similar to those of MMB were noted when wild pigs were immobilized with BAM ${ }^{\mathrm{TM}}$. Measured T of two individuals exceeded upper normal $\mathrm{T}$, but dropped within the normal range with no intervention. From a handling perspective, performing minor manipulations such as moving the body in lateral recumbency, and adjusting or handling limbs and the head elicited no responses; however, some individuals did vocalize or appear to briefly arouse when placed in dorsal recumbency and lifted by the limbs, as is often done in the field for weighing or moving. Morbidity was observed in two animals for 3 and $24 \mathrm{~h}$, respectively. We have observed similar morbidity post-BAM ${ }^{\mathrm{TM}}$ immobilization in adult wild pigs that were not part of this study (Nol, unpublished data). The dosage of $\mathrm{BAM}^{\mathrm{TM}}$ required to reach optimal immobilization in this study contained individual dosages of butorphanol and medetomidine exceeding the upper limit of published dosage ranges for swine (Plumb 2008; Carpenter 2013; Swindle and Smith 2015). Potentiation of desirable (e.g., sedation, analgesia) and undesirable (e.g., profound sedation, depression, nausea, vomiting) effects may occur when medetomidine and opiates such as butorphanol are used in combination (Plumb 2008; Girard et al. 2010). It is plausible that the higher dosages of these two drugs in the optimized BAM ${ }^{\mathrm{TM}}$ dosage produced the clinical signs of morbidity observed. Use of naltrexone to antagonize butorphanol might mitigate these effects; however, we do not routinely administer naltrexone after BAM ${ }^{\text {TM }}$ immobilization, choosing instead to allow persistence of the pharmacological effects provided by butorphanol. Potentiation of effects may occur when azaperone and atipamezole are administered, which could increase the potential for the side effects observed as well (Clark and England 1989; Sinclair 2003; Woodward 2005; Riviere and Papich 2013). Azaperone may undergo delayed absorption via sequestration in body fat, which may elicit persistence or recurrence of sedation and adverse effects (Plumb 2008). Other disadvantages associated with BAM ${ }^{\mathrm{TM}}$ may include bradycardia with/without arrhythmia and retention 
of primary or secondary drug metabolites in tissues. Like $\mathrm{MMB}, \mathrm{BAM}^{\mathrm{TM}}$ is expensive and may require controlled substance documentation. It is possible that optimizing the individual constituents in BAM ${ }^{\mathrm{TM}}$ and addition of naltrexone as a second reversal agent might result in both successful immobilization and reduced post-recovery side effects. This was beyond the scope of this study, but warrants further investigation.

Tiletamine-zolazepam/xylazine produced the least favorable statistical and observational outcomes relative to T, recovery quality, morbidity, and mortality. Importantly, individual and mean recorded Ts at the initiation of the monitoring period were at or exceeded the upper limit of normal, and remained so throughout the monitoring period in all TZX-immobilized individuals. One wild pig experienced presumed hyperthermia post-recovery but recovered without intervention, while another individual experienced documented hyperthermia and died despite interventive care. It should be noted that this animal was successfully immobilized with both MMB and $\mathrm{BAM}^{\mathrm{TM}}$ prior to administration of TZX despite gross pathologic and histopathologic findings indicating the presence of underlying pathophysiology. Recoveries were rough in all TZX-immobilized wild pigs, and behaviors and activities that likely lead to trauma and morbidity were observed for periods up to $90 \mathrm{~min}$ after animals reached level 4 recovery status. While the advantages of TZX include shorter induction time, dissociative anesthesia, analgesia, and anxiolysis, the long duration of action (1.5$5.5 \mathrm{~h}$ ), documented side effects, and our observed recoveryrelated morbidity and mortality are of concern, especially if wild pigs are to be released after immobilization for study purposes. Acute cardiopulmonary arrest and $0.35 \%$ mortality following administration of $\mathrm{TZ}$ with or without $\mathrm{K}$ or $\mathrm{X}$ in combination have been reported in domestic and feral cats (Hughes et al. 1996; Williams et al. 2002), and pre-existing pulmonary and renal disease have been causally implicated as causes of death in cats and dogs following administration of TZ (Plumb 2008). This information is of concern given that the pre-existing health status of wild pigs in the field is unknown prior to immobilization. However, given its rapid induction, low cost, and consistent availability to date, this combination may be the most practical and feasible drug combination in some situations.

Adequate sedation for safe handling was not achieved using NalMed-A at dosages approaching double the recommended starting dosage. As such, it appears that the concentrations of nalbuphine, medetomidine, and azaperone present in the pre-mixed formulation used in this study were not efficacious in wild pigs. As with BAM ${ }^{\mathrm{TM}}$, future studies designed to optimize the individual drugs may lead to developing an effective combination.

The initial $\mathrm{SpO}_{2}$ of all immobilized wild pigs, regardless of treatment, was less than or equal to $90 \%$ and improved after initiation of oxygen via nasal insufflation. Most immobilization and anesthetic agents cause CNS-derived respiratory depression, relaxation of respiratory musculature, ventilation and perfusion mismatching, and other physiological events that contribute to development of hypoxemia, leading to tissue hypoxia, myocardial ischemia, organ failure, and rhabdomyolysis (Adamson and Mills 1996; Moresco et al. 2001; Read 2003). Attempts to consistently obtain arterial blood samples were unsuccessful; however, administration of oxygen via nasal insufflation has been demonstrated effective in increasing arterial partial pressure of oxygen in other ungulate species during chemical immobilization (Paterson et al. 2009). It is therefore advisable to administer supplemental oxygen when immobilizing wild pigs regardless of the immobilization agents used.

Primary drug residues attributable to MMB administration and reversal were detected in tissues collected from one pig at day 7 post-immobilization. Residues associated with BAM ${ }^{\mathrm{TM}}$ immobilization and reversal were identified at days 3 and 7 , while residues attributable to TZX treatment and reversal were found in tissues on days 3,5 , and 7 . We did not test tissues for secondary metabolites that might have a negative impact on consumption. These results represent an area of future work, and should include extended tissue sampling time points to better assess the duration of primary drug and secondary metabolite persistence due to the potential for consumption of treated wild pigs by humans or wild animals.

Strengths of this study included our opportunity to optimize and evaluate new chemical immobilization drug combinations for wild pigs (MMB, BAM ${ }^{\mathrm{TM}}$ ) against a drug combination (TZX) commonly utilized for immobilization of wild pigs in a controlled setting, using captive reared wild pigs. Thus, while the genetics of the pigs varied, we were able to control for a number of variables including age, health status, weight, diet, environment, and other factors, which is ideal for research and development of untested products in animal species. We administered each candidate drug combination to all wild pigs in the study, and TZX to one group of randomly selected wild pigs that had been previously immobilized with $\mathrm{MMB}$ or BAM ${ }^{\mathrm{TM}}$. This allowed us to directly compare the effects of the immobilization agents on an individual and group basis, and helped control for changes in animal age and weight over time.

The fact that the wild pigs used in this study were captive reared and subjected to minimal environmental variability and stress may be viewed as behavioral confounding; however, while these pigs were habituated to human presence, routine handling was minimal. The animals, therefore, retained many of the behavioral attributes of the adult wild pigs on the premises. The age of the pigs may have resulted in some age-related bias relative to the effects of the chemical immobilization agents. We were unable to control for age; however, weight was controlled for in the statistical analysis. Despite these weaknesses, we felt that evaluating chemical immobilization agents that have limited or zero use data available in wild pigs in an environment that allowed us to control for as many factors as possible superseded the idea of attempting this study in the field where the number of confounding variables would be even greater. 
It is to be expected that adjustments in dosages will be required when immobilizing free-ranging wild pigs of variable age and size that are experiencing increased levels of stress due to capture methods, environment, reduced nutrition, compromised health, or other factors (Barasona et al. 2013), and this challenge represents an area of future study. To date, we have administered the optimized dosage and adjusted dosages of MMB (medetomidine $0.08-0.11 \mathrm{mg} / \mathrm{kg}$; midazolam $0.42-$ $0.55 \mathrm{mg} / \mathrm{kg}$; butorphanol $0.42-0.55 \mathrm{mg} / \mathrm{kg}$ ) and ВАМ ${ }^{\mathrm{TM}}$ $(1.3-1.7 \mathrm{~mL} / 45 \mathrm{~kg})$ to aggressive, fractious wild pigs ranging in weight from 37 to $170 \mathrm{~kg}$ in the field with excellent chemical immobilization results, and increased levels of safety for both the wild pigs and the human handlers (Ellis, unpublished data).

Important goals of wildlife field research include the safety of the human operators and animals, and handling of the research subjects in a manner that contributes to release of animals that are as healthy as possible in order to obtain the most relevant research data possible. Highly stressed, injured, painful, or compromised animal subjects will not produce good data. Therefore, development and use of handling methods designed to limit exertion, stress and injury, and use of reversible chemical immobilization agents that provide hypnosis, amnesia, analgesia, and muscle relaxation without undesirable changes in T, HR, R, and other physiological parameters during the peri- and post-immobilization periods are important for successful field research in wild pigs.

In conclusion, use of chemical immobilization agents that offer rapid induction, good quality immobilization, and rapid, quiet recovery with residual analgesia, and few persistent or potentially detrimental post-recovery effects should be used when field research requires safe handling and release of wild pigs back into the environment. A frequent goal of capture and release-based field research is to monitor wild pigs behaving naturally in the environment. Pharmacologically, some duration of action effects persist beyond the time that animals appear recovered from immobilization (i.e., standing, capable of movement). Therefore, post-recovery effects, and the duration of those effects, should be considered when selection of the most appropriate chemical immobilization agent is made.

Based upon our findings, we conclude that MMB appears to be the safest, most efficacious of the drug combinations compared in this study. This drug combination provides improved immobilization, recovery, and post-recovery effects relative to wild pig physiological responses, safety, morbidity, and mortality compared to BAM ${ }^{\mathrm{TM}}$ and TZX. In the event that MMB is not available for use, BAM ${ }^{\mathrm{TM}}$ constitutes our second recommendation for wild pig immobilization because physiological responses, recovery quality, and post-recovery behaviors are similar to that of MMB, and the incidence of morbidity, while troubling, is low. In comparison, TZX produces less desirable results relative to $\mathrm{T}$, and results in behaviors and activity during and after recovery for prolonged periods that contribute to trauma, injury, prolonged exertion and stress, and post-release morbidities (i.e., hyperthermia, capture myopathy), or mortality. Our observations of complications occurring during wild pig immobilization with TZX are supported by anecdotal and documented reports in the literature (Gabor et al. 1997; Sweitzer et al. 1997a, 1997b; Enqvist 2000; Wyckoff et al. 2006; Kim et al. 2007; Fenati et al. 2008; Barasona et al. 2013). If researchers intend to use TZX in the field, they must be prepared to mitigate undesirable side effects, especially when wild pigs are to be released.

Acknowledgments We would like to thank K. Held, A. Messer, M. Alfano, P. Chavez, E. Schneider, A. Kumar, and D. Gosset for assistance with animal handling, sample collection, equipment, and animal care.

Funding information This research was supported in part by the National Feral Swine Program of the U.S. Department of Agriculture.

\section{Compliance with ethical standards}

Persons interested in review or access to the study data must contact the corresponding author.

The findings and conclusions in this publication have not been formally disseminated by the U.S. Department of Agriculture and should not be construed to represent any agency determination or policy.

All applicable international, national, and/or institutional guidelines for the care and use of animals were followed.

Conflict of interest There are no potential conflicts of interest to disclose.

Open Access This article is distributed under the terms of the Creative Commons Attribution 4.0 International License (http:// creativecommons.org/licenses/by/4.0/), which permits unrestricted use, distribution, and reproduction in any medium, provided you give appropriate credit to the original author(s) and the source, provide a link to the Creative Commons license, and indicate if changes were made.

\section{References}

Adamson D, Mills C (1996) Oxygenation and ventilation. In: Duke J, Rosenberg SJ (eds) Anaesthesia secrets. Hanley and Belfus, Pennsylvania, pp 6-12

Barasona JA, López-Olvera JR, Beltrán-Beck B, Gortázar C, Vicente J (2013) Trap-effectiveness and response to tiletamine-zolazepam and medetomidine anaesthesia in Eurasian wild boar captured with cage and corral traps. BMC Vet Res 9(1):107

Carpenter JW (2013) Exotic animal formulary (ed 4). J Exotic Pet Med 22:308-309

Clark K, England G (1989) Medetomidine, a new sedative-analgesic for use in the dog and its reversal with atipamazole. J Small Anim Pract 30:343-348

Enqvist, K. E. (2000). Medetomidine/tiletamine-zolazepam and medetomidine/butorphanol/tiletamine-zolazepam: a comparison of two anesthetic regimens for surgical implantation of intraperitoneal radiotransmitters in free-ranging juvenile European wild boars (Sus scrofa scrofa). IAAAM 2000.

Federation of Animal Science Societies (2010). Guide for the care and use of agricultural animals in research and teaching. Champaign, IL

Fenati M, Monaco A, Guberti V (2008) Efficiency and safety of xylazine and tiletamine/zolazepam to immobilize captured wild boars (Sus scrofa L. 1758): analysis of field results. Eur J Wildl Res 54(2):269-274 
Gabor TM, Hellgren EC, Silvy NJ (1997) Immobilization of collared peccaries (Tayassu tajacu) and feral hogs (Sus scrofa) with Telazol ${ }^{\circledR}$ and xylazine. J Wildl Dis 33(1):161-164

Gianotti GC, Beheregaray WK, Bianchi SP, Mombach VS, Carregaro AB, Contesini EA (2010) Swine in biomedical research: normal physiological values. Acta Sci Vet 38(2):133-137

Girard NM, Leece EA, Cardwell J, Adams VJ, Brearley JC (2010) The sedative effects of low-dose medetomidine and butorphanol alone and in combination intravenously in dogs. Vet Anaesth Analg 37(1):1-6

Hannon JP, Bossone CA, Wade CE (1989) Normal physiological values for conscious pigs used in biomedical research. No. LAIR-379. Letterman Army Inst of Research Presidio of San Francisco, CA

Hodgkin BC, Burkett DE, Smith EB (1982) Noninvasive measurement of systolic and diastolic blood pressure in swine. Am J Physiol Heart Circ Physiol 242(1):H127-H130

Hughes D, Moreau RE, Overall KE, Van Winkle TJ (1996) Acute hepatic necrosis and liver failure associated with benzodiazepine therapy in six cats, 1986-1995. J Vet Emerg Crit Care 6(1):13-20

Kahn CM, Line S, Aiello S (2005) The Merck veterinary manual. Merck \& Co. Inc, Whitehouse Station, NJ

Keiter DA, Mayer JJ, Beasley JC (2016) What is in a "common" name? A call for consistent terminology for nonnative Sus scrofa. Wildl Soc Bull 40(2):384-387

Kim M, Park C, Jun M, Kim M (2007) Antagonistic effects of yohimbine in pigs anaesthetised with tiletamine/zolazepam and xylazine. Vet Rec 161(18):620-624

Ko J, Williams B, Smith V, McGrath C, Jacobson J (1993) Comparison of Telazol, Telazol-ketamine, Telazol-xylazine, and Telazol-ketamine-xylazine as chemical restraint and anesthetic induction combination in swine. Lab Anim Sci 43(5):476-480

Kumar A, Mann HJ, Remmel RP, Beilman GJ, Kaila N (2014) Pharmacokinetic study in pigs and in vitro metabolic characterization in pig- and human-liver microsomes reveal marked differences in disposition and metabolism of tiletamine and zolazepam (Telazol). Xenobiotica 44(4):379-390

Lapid R, Shilo-Benjamini Y (2015) Immobilization of captive Nubian Ibes (Capra nubiana) with butorphanol-midazolam-medetomidine or butorphanol-azaperone-medetomidine and atipamezole reversal. J Zoo Wildl Med 46(2):291-297

McCann BE, Smyser TJ, Schmit BS, Newman RA, Piaggio AJ, Malek MJ, Swafford SR, Sweitzer RA, Simmons RB (2018) Molecular population structure for feral swine in the United States. J Wildl Manag 82(4):821-832

Mich PM, Wolfe LL, Sirochman TM, Sirochman MA, Davis TR, Lance WR, Miller MW (2008) Evaluation of intramuscular butorphanol, azaperone, and medetomidine and nasal oxygen insufflation for the chemical immobilization of white-tailed deer, Odocoileus virginianus. J Zoo Wildl Med 39(3):480-487

Miller BF, Osborn DA, Lance WR, Howze MB, Warren RJ, Miller KV (2009) Butorphanol-azaperone-medetomidine for immobilization of captive white-tailed deer. J Wildl Dis 45(2):457-467

Moresco A, Larsen RS, Sleeman JM, Wild MA, Gaynor JS (2001) Use of naloxone to reverse carfentanil citrate-induced hypoxemia and cardiopulmonary depression in Rocky Mountain wapiti (Cervus elaphus nelsoni). J Zoo Wildl Med 32(1):81-89

Morgaz J, Navarrete R, del Mar Granados M, Gómez-Villamandos RJ (2015) Swine model in transplant research: review of anaesthesia and perioperative management. World J Anes 4(3):73-82

Nolte D, Anderson W (2015) Collaborating to halt feral swine damage. Wildl Prof 9:56-59

Paterson JM, Caulkett NA, Woodbury MR (2009) Physiologic effects of nasal oxygen or medical air administered prior to and during carfentanil-xylazine anesthesia in North American elk (Cervus canadensis manitobensis). J Zoo Wildl Med 40(1):39-50
Paul E, Sikes RS, Beaupre SJ, Wingfield JC (2016) Animal welfare policy: implementation in the context of wildlife research - policy review and discussion of fundamental issues. ILAR J 56(3):312-334

Plumb DC (2008) Plumb's vet drug handbook: desk edition. Blackwell, Ames, IA

Price EO (1984) Behavioral aspects of animal domestication. Q Rev Biol 59(1):1-32

Read MR (2003) A review of alpha2 adrenoreceptor agonists and the development of hypoxemia in domestic and wild ruminants. J Zoo Wildl Med 34(2):134-138

Riviere, J. E. and M. G. Papich (2013). Veterinary pharmacology and therapeutics, John Wiley \& Sons

Sakaguchi M, Nishimura R, Sasaki N, Ishiguro T, Tamura H, Takeuchi A (1992) Sedative effects of medetomidine in pigs. J Vet Med Sci 54(4):643-647

Sinclair MD (2003) A review of the physiological effects of $\alpha 2$-agonists related to the clinical use of medetomidine in small animal practice. Can Vet J 44(11):885

Sipos W, Wiener S, Entenfellner F, Sipos S (2013) Physiological changes of rectal temperature, pulse rate and respiratory rate of pigs at different ages including the critical peripartal period. Vet Med Austria 100(3):96

Smith A, Hawkins P (2016) Good science, good sense and good sensibilities: the three Ss of Carol Newton. Animals 6(11):70

Spraker TR (1982) An overview of the pathophysiology of capture myopathy and related conditions that occur at the time of capture of wild animals. Chem Immobil N Am Wildl 83:118

Swart JA (2004) The wild animal as a research animal. J Agric Environ Ethics 17(2):181-197

Sweitzer RA, Ghneim GS, Gardner IA, Vuren DV, Gonzales BJ, Boyce WM (1997a) Immobilization and physiological parameters associated with chemical restraint of wild pigs with Telazol® and xylazine hydrochloride. J Wildl Dis 33(2):198-205

Sweitzer RA, Gonzales BJ, Gardner IA, Van Vuren D, Waithman JD, Boyce WM (1997b) A modified panel trap and immobilization technique for capturing multiple wild pig. Wildl Soc Bull 25(3):699-705

Sweitzer RA, McCann BE, Loggins RE, Simmons RB (2016) Mitochondrial DNA perspectives on the introduction and spread of wild pigs in California. Calif Fish Game 101(2):131-145

Swindle, M. M. and A. C. Smith (2015). Swine in the laboratory: surgery, anesthesia, imaging, and experimental techniques, CRC press

William MR (1959) The principles of humane experimental technique. ALTEX 3:94

Williams LS, Levy JK, Robertson SA, Cistola AM, Centonze LA (2002) Use of the anesthetic combination of tiletamine, zolazepam, ketamine, and xylazine for neutering feral cats. JAVMA 220(10):14911495

Wolfe LL, Goshorn CT, Baruch-Mordo S (2008) Immobilization of black bears (Ursus americanus) with a combination of butorphanol, azaperone, and medetomidine. J Wildl Dis 44(3):748-752

Wolfe LL, Fisher MC, Davis TR, Miller MW (2014a) Efficacy of a lowdosage combination of butorphanol, azaperone, and medetomidine (BAM) to immobilize Rocky Mountain elk. J Wildl Dis 50(3):676680

Wolfe LL, Lance WR, Smith DK, Miller MW (2014b) Novel combinations of nalbuphine and medetomidine for wildlife immobilization. J Wildl Dis 50(4):951-956

Wolfe LL, Wood ME, Nol P, McCollum MP, Fisher MC, Lance WR (2017) The efficacy of nalbuphine, medetomidine, and azaperone in immobilizing American bison (Bison bison). J Wildl Dis 53(2):304-310

Wolff P (2009) Exotic Suid anesthesia and analgesia. Proc AAZV/ AAWV Conf, Tulsa, OK

Woodward K (2005) Veterinary pharmacovigilance. Part 4. Adverse reactions in humans to veterinary medicinal products. J Vet Pharm Ther 28(2):185-201 
Wyckoff, A. C., S. E. Henke, T. Campbell and K. C. VerCauteren (2006). Is trapping success of feral hogs dependent upon weather conditions? Proc Vertebr Pest Conf

Wyckoff AC, Henke SE, Campbell TA, Hewitt DG, VerCauteren KC (2009) Feral swine contact with domestic swine: a serologic survey and assessment of potential for disease transmission. J Wildl Dis 45(2):422-429

Publisher's note Springer Nature remains neutral with regard to jurisdictional claims in published maps and institutional affiliations. 